Voix et Images

\title{
EXPÉRIENCES CONTEMPORAINES DU TEMPS DANS LES FICTIONS QUÉBÉCOISES
}

\section{Manon Auger et Marion Kühn}

Volume 44, numéro 2 (131), hiver 2019

Expériences contemporaines du temps dans les fictions québécoises

URI : https://id.erudit.org/iderudit/1059511ar

DOI : https://doi.org/10.7202/1059511ar

Aller au sommaire du numéro

Éditeur(s)

Université du Québec à Montréal

ISSN

0318-9201 (imprimé)

1705-933X (numérique)

Découvrir la revue

Citer ce document

Auger, M. \& Kühn, M. (2019). EXPÉRIENCES CONTEMPORAINES DU TEMPS

DANS LES FICTIONS QUÉBÉCOISES. Voix et Images, 44(2), 7-12.

https://doi.org/10.7202/1059511ar d'utilisation que vous pouvez consulter en ligne.

https://apropos.erudit.org/fr/usagers/politique-dutilisation/ 


\title{
EXPÉRIENCES CONTEMPORAINES DU TEMPS DANS LES FICTIONS QUÉBÉCOISES
}

\author{
$+++$ \\ MANON AUGER \\ Université du Québec à Montréal \\ MARION KÜHN \\ Chercheuse indépendante
}

Dans la foulée des travaux de Paul Ricœur ${ }^{1}$, de Pierre Nora $^{2}$, mais surtout de François Hartog $^{3}$, on reconnaît de plus en plus une modification de l'expérience du temps en régime contemporain. En effet, que l'on évoque le "présentisme» théorisé par Hartog - «ce progressif envahissement de l'horizon par un présent de plus en plus gonflé, hypertrophié ${ }^{4} »-$, «le devoir de mémoire» ou la «fin des idéologies», marquée historiquement par la chute du mur de Berlin en 1989, c'est toujours peu ou prou à une césure dans l'ordre du temps qu'on se réfère ou, du moins, à un changement de paradigme qui aurait induit un nouvel «être du temps ${ }^{5}$ », celui que Jean-François Hamel nomme le «bègue incertain» et qui, «après le maître autoritaire et le maigre inquiétant ${ }^{6}$ », «balbutie le temps plutôt qu'il n'en énonce la loi, [...] en cherche la saveur et le savoir sans parvenir à en énoncer sans heurts l'articulation secrète ${ }^{7} »$.

Ainsi, le présentisme ancrerait l'individu contemporain dans un présent à la fois inquiet et omniprésent, tout en lui faisant craindre l'avenir (no future) et «ruminer un passé qui ne passe pas ${ }^{8}$ ». Dès lors, les dispositifs juridiques de précaution puisant dans le "principe responsabilité ${ }^{9}$ », le «boom de la mémoire ${ }^{10}$ », voire

1 Paul Ricœur, La mémoire, l'histoire, l'oubli, Paris, Éditions du Seuil, coll. «Ordre philosophique», 2000, 675 p.

2 Pierre Nora (dir.), Les lieux de mémoire, avec la collaboration de Charles-Robert Ageron, Paris, Gallimard, coll. «Bibliothèque illustrée des histoires», 1984-1992, 3 tomes en 7 volumes.

3 François Hartog, Régimes d'historicité. Présentisme et expériences du temps, Paris, Éditions du Seuil, coll. «La librairie du xxI siècle», 2003, 257 p.

4 Ibid., p. 125.

5 Jean-François Hamel, «Le maître, le maigre et le bègue. Avant-propos», Jean-François Hamel et Virginie Harvey (dir.), Le temps contemporain : maintenant, la littérature, Montréal, Université du Québec à Montréal/ Figura, coll. «Figura», 2009, p. 12.

6 Le «maître autoritaire» renvoie à ce qu'Hartog appelle le régime ancien d'historicité (Hamel: «l'histoire du passé était dépositaire d'une sagesse fournissant des règles de vie», ibid., p. 13), tandis que le «maigre inquiétant» renvoie à ce qu'Hartog appelle le régime moderne d'historicité (Hamel: «rien de ce qui fut ne permettait désormais de réfléchir ce qui advient et de présager ce qui sera», ibid., p. 14).

7 Ibid., p. 15.

8 François Hartog, Régimes d’historicité, p. 206.

9 Hans Jonas, Le principe responsabilité: une éthique pour la civilisation technologique, traduit de l'allemand par Jean Greisch, Paris, Éditions du Cerf, 1990, 336 p.

10 Andreas Huyssen, Present Pasts: Urban Palimpsests and the Politics of Memory, Stanford, Stanford University Press, 2003, p. 18. 
la «commémorationnite ${ }^{11}$ » qui accompagnent le présentisme sont, pour beaucoup d'observateurs, autant de symptômes d'un nouveau rapport au temps marqué par la "crise de l'avenir ${ }^{12}$ » comme par la «fin des grands récits ${ }^{13}$ ». Retranché dans l'espace privé et dépossédé de ses repères anciens face à l'éclatement d'une réalité devenue ininterprétable, l'individu contemporain non seulement n'articulerait plus passé, présent et avenir comme le faisait l'individu moderne, mais il serait, de plus, le témoin ou la victime tant des «abus de (la) mémoire ${ }^{14}$ » que d'une société d' «après $l^{\prime}$ Histoire ${ }^{15}$ ». C'est donc dire, d'une part, que l'expérience contemporaine du temps est intimement liée à la question de la mémoire et à celle de l'Histoire - sans pouvoir s'y résumer complètement - et que, d'autre part, le sentiment d'un "venir après " teinte fortement les expériences personnelles et collectives du temps dans le monde occidental. On pourra même soutenir que, dans la mesure où le passé est «devenu inintelligible» et que le futur prend «au mieux la forme d'une répétition intensifiée du présent, au pire d'une catastrophe annoncée marquant la fin de l'humanité», l'individu contemporain est prisonnier d'un présent qui lui rappelle sans cesse qu'il est réduit à «sa propre condition d'être temporel et mortel ${ }^{16} »$. Dans ces circonstances, les expériences contemporaines du temps ne peuvent en effet qu'être vertigineuses, troublées, «bégayantes»...

En un certain sens, la littérature contemporaine, du moins en France, participe de ce rapport complexe au temps; elle se fait, entre autres, l'écho d'un temps en souffrance où le passage des générations ne s'accompagne pas d'une transmission, qu'il convient donc d'inventer si ce n'est de susciter par l'écriture. Il n'y a qu'à songer, pour s'en convaincre, aux récits de filiation ${ }^{17}$ qui présentent, souvent sur le mode désenchanté, une tentative de renouer avec ses ascendants, ou encore aux romans de la mémoire ou de la "post-mémoire ${ }^{18}$ » qui soulèvent les enjeux éthiques et identitaires de la remémoration ou de la commémoration. On peut songer, également, au nouveau roman historique (parfois qualifié de postmoderne) qui tente de montrer une mise en récit problématique de l'Histoire; le récit y paraît alors comme la tentative aussi subjective que sélective de recréer par les mots ce qui n'est plus, et

11 François Chenet, Le temps : temps cosmique, temps vécu, Paris, Armand Colin, coll. «U. Philosophie», 2000, p. 155.

12 Krzysztof Pomian, «La crise de l'avenir», Le Débat, nº 7, 1980, p. 5-17.

13 Sur le sujet, voir: Jean-François Lyotard, La condition postmoderne. Rapport sur le savoir, Paris, Éditions de Minuit, coll. «Critique», 1979, 109 p.

14 Tzvetan Todorov, Les abus de la mémoire, nouvelle édition, Paris, Arléa, coll. «Arléa-poche», 2015 [1995], $59 \mathrm{p}$.

15 Philippe Muray, Après l'Histoire I. Essai, Paris, Les Belles Lettres, 1999, 275 p.

16 E.-Martin Meunier et Joseph Yvon Thériault (dir.), «Introduction. Le "moment mémoire" à l'heure du présentisme contemporain", Les impasses de la mémoire. Histoire, filiation, nation et religion, Montréal, Fides, 2007, p. 25.

17 Voir Dominique Viart, «Filiations littéraires», Jan Baetens et Dominique Viart (dir.), États du roman contemporain, actes du colloque de Calaceite, Fondation Noesis, 6-13 juillet 1996, Paris, Lettres modernes/Minard, coll. "Revue des lettres modernes. Écritures contemporaines», 1999, p. 115-139, et Laurent Demanze, Encres orphelines. Pierre Bergounioux, Gérard Macé, Pierre Michon, Paris, José Corti, coll. «Les essais», 2008, $403 \mathrm{p}$.

18 Marianne Hirsch, Family Frames. Photography, Narrative, and Postmemory, Cambridge, Harvard University Press, 1997, $304 \mathrm{p}$. 
ce, à partir d'un présent tout aussi difficile à saisir. L'écrivain français Olivier Rolin l'exprime d'ailleurs de façon très nette:

Ce que ma génération aura vécu de plus profond et durable, en définitive, c'est le passage d'une structure du temps où les dimensions du futur et du passé étaient très fortement marquées à un nouveau paradigme temporel où ces dimensions prospectives et rétrospectives se sont presque effacées au profit d'un présent hypertrophié. Et au centre de la bulle immense du «temps réel», sillonné de l'éphémère de trajectoires désordonnées, il y a (il y aurait) le point fixe du moi, et de ce qu'il comporte de plus patent: le corps. Tel est le dispositif contemporain ${ }^{19}$.

Le "moi», le «corps», les perceptions... C'est peut-être en effet toujours de soi dont il s'agit désormais en littérature, non pas exclusivement en raison d'un narcissisme envahissant, mais bien parce que cela demeure le seul point nodal à partir duquel chaque écrivain peut désormais regarder le monde et tenter $\mathrm{d}^{\prime} \mathrm{y}$ comprendre quelque chose $^{20}$.

Ce mouvement - lui-même très actuel - qui pousse à réfléchir (sur) notre rapport au temps tout autant qu'à la mémoire et à l'Histoire paraît également occuper et préoccuper la fiction québécoise des deux dernières décennies - mais selon une concordance qu'il reste encore à vérifier. Pensons, par exemple, au motif de la filiation, point de départ pour la conception d'un imaginaire du temps ou d'une opposition de temporalités dans Les taches solaires de Jean-François Chassay (Boréal, 2006) et dans Les fossoyeurs d'André Lamontagne (Éditions David, 2010). Pensons également à l'arrivée de personnages-enquêteurs, obsédés par leur quête historique au point d'oublier le présent, dans des romans comme La constellation du Lynx de Louis Hamelin (Boréal, 2010), Il pleuvait des oiseaux de Jocelyne Saucier (XYZ éditeur, 2011) ou L'année la plus longue de Daniel Grenier (Le Quartanier, 2015). Pensons, finalement, à ces récits de mémoire ou de "post-mémoire» que sont Artéfact de Carl Leblanc (XYZ éditeur, 2012) ou Le ciel de Bay City de Catherine Mavrikakis (Héliotrope, 2008), deux cas de figure d'une mise en scène de la mémoire de la Shoah qui fait irruption, dans le premier cas, dans un présent saturé de commémorations et, dans le deuxième, dans un présent hanté par le passé refoulé. Ainsi, ces expériences du temps, souvent vécues de façon individuelle, semblent être le reflet d'une conscience collective marquée tout comme en France au sceau du présentisme, mais pourraient bien aussi - c'est une hypothèse - constituer des tentatives pour y échapper, pour penser autrement le rapport au temps, à la mémoire et à l'histoire, depuis un point de vue spécifique - celui de l'écriture - québécois.

19 Olivier Rolin, «Un écrivain doit-il aimer son époque?», Bric et broc, Paris, Verdier, 2011, p. 68.

20 C'est une hypothèse que propose Daniel Letendre, dans son essai récent sur le récit français contemporain: «Difficile pour un sujet qui a le sentiment d'habiter un présent non conforme à ses aspirations, guidé par des principes qu'il ne reconnaît pas et organisé selon un ordre du temps qui n'est pas le sien, de se sentir en contemporanéité avec ce monde. La seule solution qu'il peut envisager pour pallier cet anachronisme est de s'accorder à lui-même, de fonder en lui et à partir de lui l'ordre temporel avec lequel il peut être contemporain. » Daniel Letendre, Pratiques du présent. Le récit français contemporain et la construction narrative du temps, Montréal, Presses de l’Université de Montréal, coll. «Espace littéraire», 2018, p. 90. 
C'est précisément la complexité de ces expériences contemporaines du temps, telles qu'elles se déploient dans et par les fictions québécoises, que nous avons voulu explorer dans ce dossier. La modification du rapport au temps, observée par les spécialistes des sciences humaines, ouvre-t-elle sur de nouveaux espaces, de nouveaux lieux, de nouvelles conceptions du présent, du passé et du futur? Autrement dit: les fictions, avec les moyens qui leur sont propres, reconduisent-elles simplement les tristes constats actuels ou, au contraire, amènent-elles à penser différemment cet «être du temps» qui serait le propre de la période contemporaine? Bien sûr, les réponses que l'on pourra fournir à ces questions, ici et au fil des articles, seront forcément partielles, mais, alors que nombre de spécialistes français, dont Dominique Viart ${ }^{21}$, Laurent Demanze ${ }^{22}$ et Gianfranco Rubino ${ }^{23}$, semblent voir des échos très nets entre les propos des spécialistes de sciences humaines et la littérature française contemporaine, nous avons choisi pour notre part de ne pas tenir ces constats pour acquis en ce qui concerne spécifiquement la littérature québécoise. Du moins préférons-nous voir ce portrait présentiste comme une première étape, une esquisse fort utile qui nous permet de mieux comprendre notre contemporain de manière générale ${ }^{24}$, nous aidant à mieux réfléchir, ensuite, à la manière dont la littérature, à son tour, problématise la question du temps. Car, comme le constate Hamel, si le portrait présentiste est sombre, il n'en demeure pas moins que "c'est l'inventivité de la littérature qui se trouve [dès lors] mise à l'épreuve et, du coup, relancée ${ }^{25} »$. Autrement dit, si l'avenir de toute chose est incertain, le présent de la littérature n'abdique pas pour autant et cherche parfois, comme le propose Karen S. McPherson à propos des écritures féministes ${ }^{26}$, à profiter du chaos pour «amorcer un nouveau commencement des temps ${ }^{27}$ ».

21 Viart soutient que les travaux des historiens sur les régimes d'historicité ont permis de donner une «orchestration décisive» aux travaux menés par les théoriciens de la littérature française contemporaine. Voir «La littérature, l'histoire, de texte à texte», Gianfranco Rubino et Dominique Viart (dir.), Le roman français contemporain face à l'Histoire. Thèmes et formes, Macerata (Italie), Quodlibet Studio, 2014, p. 39-40.

22 Dans Encres orphelines, par exemple, Demanze parle d'une «douloureuse mélancolie, qu'agite sans cesse la conscience blessée d'un passé perdu». Laurent Demanze, Encres orphelines, p. 9.

23 Rubino, qui s'intéresse beaucoup à l'écriture du passé dans ses différents travaux, dira par exemple: «Loin d'exclure le monde d'aujourd'hui, ce va-et-vient passé/présent semble le concerner de près. À condition de comprendre le statut mélangé d'un présent qui ne se suffit pas à lui-même et qui, faute de projection dans l'avenir, empiète continuellement sur un passé dont plusieurs symptômes culturels montrent l'emprise perturbante.» Gianfranco Rubino, «La hantise du passé», Dominique Viart et Laurent Demanze (dir.), Fins de la littérature, t. II : Historicité de la littérature contemporaine, Paris, Armand Colin, coll. «Recherches», 2012, p. 172.

24 Pour une perspective sociologique sur le rapport à l'histoire, à la mémoire et au temps au Québec, on consultera: E.-Martin Meunier et Joseph Yvon Thériault (dir.), Les impasses de la mémoire. Les auteurs évoquent un «moment mémoire», "qui a pris d'abord son envol dans l'historiographie pour s'étendre graduellement à d'autres disciplines (philosophie, sociologie, études littéraires, etc.), mais qui fut aussi présent dans l'espace politique». Ils ajoutent: «Le moment mémoire est une réponse partielle et maladroite à la crise de l'avenir qui a secoué et qui secoue encore nos sociétés. On peut notamment croire que le moment mémoire est venu se glisser à la place laissée vacante par l'utopie et il en aurait rempli un peu la fonction, comme à son corps défendant.» (p. 9-10)

25 Jean-François Hamel, «Le maître, le maigre et le bègue», p. 15.

26 Voir Karen S. McPherson, Archaeologies of an Uncertain Future. Recent Generations of Canadian Women Writing, Montréal, McGill-Queen's University Press, 2006, 303 p.

27 Ibid., p. 7. McPherson cite Louky Bersianik, La main tranchante du symbole. Textes et essais féministes, Montréal, Éditions du Remue-ménage, coll. «Itinéraires féministes», 1990, p. 267. 
Cela étant, il faut reconnaître que la question des expériences du temps ne se laisse pas si aisément saisir, et nos collaborateurs et nous avons dû emprunter ici des voies détournées pour en apprécier les différentes nuances: celle de l'imaginaire western pour Andrée Mercier, de la mémoire pour Marion Kühn, de l'histoire chez David Bélanger, de la biographie chez Pierre-Olivier Bouchard et, finalement, de la figure du héros chez Manon Auger.

Ainsi, dans son article, Andrée Mercier s'intéresse au rapport au temps véhiculé par l'appropriation de l'imaginaire western dans Griffintown de Marie-Hélène Poitras et À la recherche de New Babylon de Dominique Scali. Plus spécifiquement, elle analyse l'interaction des figures de la répétition et de la légende qui traduisent la « temporalité complexe» inhérente à cet imaginaire afin de distinguer la manière dont «deux ordres du temps se confrontent» au sein de ces romans. Exposant comment Griffintown crée un «hors temps épique» tandis qu'À la recherche de New Babylon met en place le «sentiment d'urgence» d'une course perdue à l'avance, elle détecte aussi de possibles chemins qui permettent aux personnages de «sortir le présent de la répétition»".

Marion Kühn s'intéresse pour sa part à la «mémoire transculturelle» dans les écrits d'Anaïs Barbeau-Lavalette, de Judy Quinn et de Carole David. Amalgamant histoires familiales et histoires des «autres» à même la mémoire au présent des différents personnages, ces récits mettent de l'avant la «dimension humaine de la mémoire qui traverse les frontières", mais c'est surtout leurs mises en récit particulières qui permettent une saisie différente de la relation entre passé et présent. Tantôt vécu comme un "trou à combler», tantôt comme une fiction, le passé se révèle éminemment stratifié, mais, dans tous les cas, le récit doit le faire advenir pour soulager le présent de sa mémoire.

À partir d'une réflexion sur l'histoire «qui ne suffit plus» pour comprendre le présent, David Bélanger s'intéresse au passé organiquement vécu par différents personnages de la littérature québécoise contemporaine (chez Raymond Bock et Éric Dupont principalement). Dans plusieurs œuvres récentes, constate-t-il, se retrouve ce «geste contemporain particulier qui consiste à vivre le temps plutôt qu'à édifier l'histoire ou à s'y inscrire». C'est un peu comme si l'Histoire - et en particulier l'histoire nationale - ne suffisait plus pour assurer une continuité entre passé et présent, mais, tandis que certains se réjouissent d'être débarrassés de la téléologie de l'histoire, d'autres vivent cette «rupture avec la rupture» de manière plus nostalgique.

Un autre récit d'histoire, celui de la littérature québécoise, est au centre de l'article de Pierre-Olivier Bouchard, qui propose deux lectures d'Arthur Buies, chevalier errant de Micheline Morisset. Afin de mettre en lumière la complexité du rapport au temps dans cette fiction biographique, il la présente d'abord comme une «fiction présentiste», pour montrer, par la suite, que «l'œuvre porte en elle une conception du temps qui lui est propre», notamment par son regard optimiste sur l'avenir des lettres québécoises qui constitue une «réécriture de l’histoire littéraire» autour de la «figure structurante» de Buies.

La mise en scène de personnages historiques intéresse aussi Manon Auger. Dans sa contribution, elle scrute la possibilité de l'héroïsme en régime présentiste qui ne permet que des "gestes héroïques en minuscule», des gestes qui relèvent, 
constate-t-elle, d'une «expérience de l'immobilité». À l'aide de l'exemple des deux antagonistes du roman Du bon usage des étoiles de Dominique Fortier, John Franklin et son second, Francis Crozier, elle oppose le rapport au temps du héros moderne visant à «imprégner sa marque sur l'Histoire» à celui de son pendant contemporain qui, lui, agit sur son présent en faisant preuve d'ouverture d'esprit et d'introspection, bref, d'une «sensibilité contemporaine» qui lui permet de "créer de nouveaux points de repère». S'appuyant sur Immobile de Ying Chen et La respiration du monde de Marie-Pascale Huglo, elle met ensuite en lumière la solitude et le sacrifice du héros contemporain, conscient des failles de l'ordre du temps dans lequel il est contraint d'évoluer, mais auquel il résiste dans une immobilité qui n'est peut-être qu'apparente.

Il fut un temps où l'on croyait en l'immortalité de l'âme, ce qui garantissait un sens à la vie sur terre et un futur marqué par la promesse d'une récompense. Cette croyance a peu à peu été remplacée, aux $\mathrm{xIx}^{\mathrm{e}}$ et $\mathrm{xx}^{\mathrm{e}}$ siècles, par celle de la "vocation», qui garantissait à son tour un sens aux destinées, un point d'arrivée. Aujourd'hui, ces repères ne suffisent plus, et le temps est le plus souvent le lieu d'une expérience anxiogène, non seulement en raison du retour quasi obsessionnel du passé ou de «l'écrasement du temps entraîné par la pression de l'urgence et de la servitude de l'instant conjugués ${ }^{28}$ » qui caractérisent le rapport au temps présentiste, mais aussi en raison de l'absence d'une transcendance qui donnerait sens au présent et au futur.

Ce sens, nombreux sont ceux qui le recherchent à travers l'écriture et la littérature: c'est le cas, on le verra, de personnages fictifs comme Francis Crozier (Fortier) et le révérend Aaron (Scali), mais aussi d'écrivaines comme Anaïs Barbeau-Lavalette, Micheline Morisset ou Judy Quinn. Car si «l'histoire ne suffit plus» pour assurer une continuité entre passé et présent, comme le propose Bélanger, la littérature, elle, permet de mettre au jour ce passé qui fait retour dans une mémoire à la fois affective et corporelle et qui empêche d'habiter le présent. L'écriture ne sauve pas tout, certes, mais elle offre à tout le moins la possibilité d'apprivoiser un peu le temps, de l'accorder à l'organicité de la vie elle-même et, surtout, de le rendre un peu moins bègue.

28 François Chenet, Le temps: temps cosmique, temps vécu, p. 154. 